\title{
Extra Skeletal Myxoid Chondrosarcoma of the Foot: A Case Report
}

Sidki Sanaa ${ }^{1,2}$, Zongo Pawendtaoré Esdras ${ }^{1,2^{*}}$, Benchakroun Nadia, ${ }^{1,2}$ Chekrine Tarek ${ }^{1,2}$, Bourhafour Mouna ${ }^{1,2}$, Bouchbika Zineb $^{1,2}$, Jouhadi Hassan ${ }^{1,2}$, Tawfiq Nezha ${ }^{1,2}$, Sahraoui Souha ${ }^{1,2}$, Benider Abdellatif ${ }^{1,2}$

\footnotetext{
${ }^{1}$ Department of Radiotherapy and Oncology CHU Ibn Rochd Casablanca, Morocco

${ }^{2}$ Faculty of Medicine and Pharmacy, Hassan II University Casablanca, Morocco
}

DOI: $10.36347 /$ simcr.2022.v10i01.007

| Received: 10.12.2021 | Accepted: 15.01.2022 | Published: 24.01.2022

*Corresponding author: Zongo Pawendtaoré Esdras

Department of Radiotherapy and Oncology CHU Ibn Rochd Casablanca, Morocco

Abstract

Introduction: Extra skeletal myxoid chondrosarcoma is a rare soft tissue tumor. It is a low-grade sarcoma, arising mainly in the deep tissues and musculature of the extremities, with a histological resemblance to its bony counterpart. It accounts for less than $3 \%$ of soft tissue sarcomas, and localizes mainly in the upper extremity, shoulder, neck, orbit but also intra-abdominally. It is a tumor with a high metastatic potential and an unfavorable long-term prognosis. Extra skeletal myxoid chondrosarcoma is a tumor occurring in middle-aged patients regardless of gender or race. Treatment is multimodal including surgery, radiation therapy and chemotherapy. Case presentation: A 37-year-old female patient consulted for a subcutaneous swelling on the sole of the left foot opposite the 5th toe; evolving for approximately six years. The clinical examination and the paraclinical work-up revealed a mass that encompassed the diaphysis of the 5th metatarsal with cortical lysis opposite. This mass arrived medially at the contact of the 4th metatarsal without cortical lysis. The extension work-up was unremarkable. The histological, immunohistochemical and molecular biology examination of the surgical biopsy concluded to an extra skeletal myxoid chondrosarcoma. Polychemotherapy with andriamycin and Ifosfamide, surgery and radiotherapy at a dose of 50Gy was performed. Conclusion: Rare tumor and of difficult diagnosis, the key of the diagnosis lies in molecular biology. Survival is often prolonged despite its local aggressiveness.

Keywords: Extra skeletal, Myxoid, Chondrosarcoma, Foot.

Copyright () 2022 The Author(s): This is an open-access article distributed under the terms of the Creative Commons Attribution 4.0 International License (CC BY-NC 4.0) which permits unrestricted use, distribution, and reproduction in any medium for non-commercial use provided the original author and source are credited.

\section{INTRODUCTION}

Extra skeletal myxoid chondrosarcoma (ESMC), also known as chordoid sarcoma, is a rare soft tissue tumor first described in 1953 by Stout and Verner [1], but it was not until 1972 that Enzinger an Shiraki [2] defined the myxoid variant as a distinct clinicopathologic entity [3]. Extra skeletal myxoid chondrosarcoma is a low-grade soft tissue sarcoma, occurring primarily in the deep tissues and musculature of the extremities, which histologically resembles its bone counterpart [3]. It accounts for less than $3 \%$ of soft tissue sarcomas [4]. Other locations such as upper extremity, shoulder, neck, orbit and intra-abdominal wall have also been reported [5, 6]. It has an indolent natural history characterized by the development of metastases, mainly to the lung [7]. According to the literature, this tumor has an unfavorable long-term prognosis [8]. It is a tumor that frequently occurs in middle-aged patients without any predilection for gender or race [3]. Histologically, characterized by well-circumscribed tumor nodules separated by variable amounts of mucoid material. Individual cells are characteristic of chondroblasts, uniform in size and shape, and are arranged in short anastomosing strands and cords resulting in a spider web appearance [3]. Traditionally, treatment of patients with extraskeletal myxoid chondrosarcoma has included surgical resection with or without radiation therapy [3]. Patients who are candidates for systemic therapy should preferably be treated according to investigative protocols [3]. Firstline chemotherapy has not been proven $[9,10]$. We report the case of a foot location in a 37-year-old woman.

\section{Case Presentation}

Mrs M. N, 37 years old and without any particular pathological history, consulted for a subcutaneous swelling on the sole of her left foot opposite the 5th toe, which had been evolving for about six years. The MRI of the left foot showed the presence of a mass in the sole of the foot with hypo T1 signal, hyper T2 signal and STIR, with lobulated contours, 
strongly enhanced after injection of gadolinium with delimitation of striations in hypo signal. This mass measures $39 \times 20 \times 24 \mathrm{~mm}$ in long axis, it encompasses the diaphysis of the 5th metatarsal with cortical lysis opposite and hypo signal of the cancellous bone (Figure 1). It extended to the subcutaneous fat opposite and arrived medially at the contact of the 4th metatarsal without detectable cortical lysis. The extension workup was negative. A surgical excisional biopsy was performed. Histological examination showed a nodular and lobulated tumor proliferation. The lobules are separated by fibrous tracts of variable thickness. Within the lobules, the tumor cells are grouped in small and large clusters without being cohesive; these clusters are bathed in a myxoid substance. The cells have ovoid nuclei with fine chromatin, sometimes with a small nucleolus, the cytoplasm is eosinophilic or clear, without PAS+ crystals. Some nuclei are grooved or notched (Figure 2). An immunohistochemical study

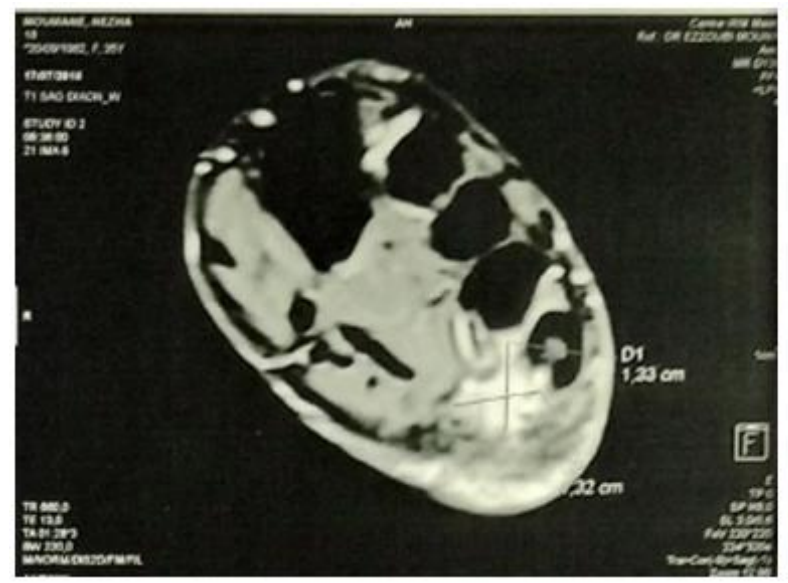

Fig-1: MRI of the left foot: A mass in the sole of the foot of $39 \times 20 \times 24 \mathrm{~mm}$ in long axis, it encompasses the diaphysis of the 5th metatarsal with cortical lysis opposite.

\section{DISCUSSION}

Extraskeletal myxoid chondrosarcoma is an unusual soft tissue sarcoma that has been recognized for some time. Originally, it was called chordoid sarcoma because it histologically resembled a chordoma. Various reports and reviews describing extraskeletal myxoid chondrosarcoma (chordoid sarcoma) have been published [2, 7, 11] including a report by Weiss in 1976 [11] that provided evidence based on electron microscopic studies for the cartilaginous differentiation of this lesion. Thus, the former chordoid sarcoma was dropped, and the tumor has since been called extraskeletal myxoid chondrosarcoma [1].

Clinically, it occurs in adults around the age of 50 with a male predominance and a sex ratio of 2:1 [1]. However, the peak frequency is in the third decade when it affects the central nervous system and in the fifth decade for soft tissue or muscle localizations [12]. Our observation does not correspond to the latter peak. Its location is in fact ubiquitous, but a location in the thigh and knee should attract attention. Other possible

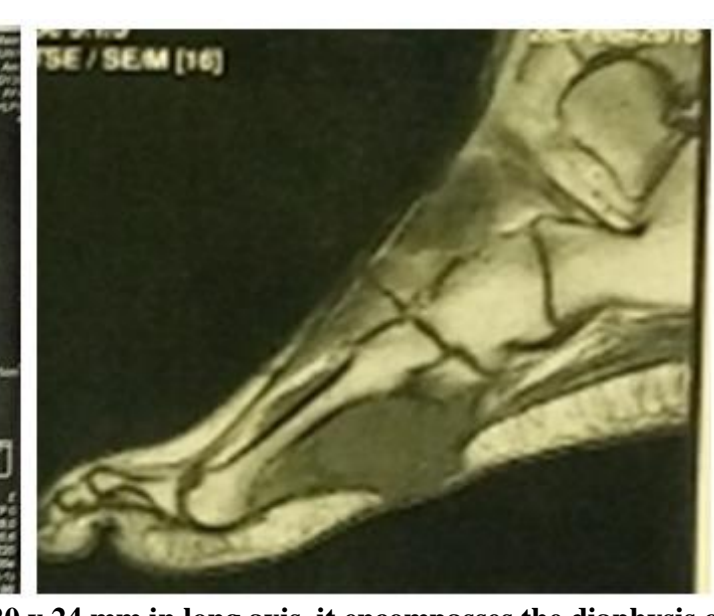

showed tumor cells diffusely expressing EMA and focally Synaptophysin without expressing Cytokeratin AE1/AE3, CD34, PS100, HMB45, Chromogranin A and Smooth muscle Actin. Oncologic cytogenetic examination showed a rearrangement of the EWS gene in $80 \%$ of the nuclei analyzed. A rearrangement of the $22 \mathrm{q} 12$ band (EWS) was also observed. The translocation $\mathrm{t}(19 ; 22)(\mathrm{q} 22 ; \mathrm{q} 12)$ is indeed reported. The histological, immunohistochemical and molecular biology analysis concluded to an extra skeletal myxoid chondrosarcoma. The patient underwent neoadjuvant polychemotherapy with andriamycin and Ifosfamide for 18 weeks from D1 to D21, followed by complete excision and then adjuvant chemotherapy with two courses of Ifosfamide alone and radiotherapy at a dose of 50Gy. At 24 months of follow-up, no local recurrence or distant metastases were observed.

\section{FIGURES}


lobules and/or trabeculae. The tumor cells are monotonous, medium-sized, mostly round or slightly elongated, with clear or eosinophilic cytoplasm. Rhabdoid inclusions are seen in $10 \%$ of cases. The nucleus is usually small, reniform or ovoid, with fine chromatin, revealing a small nucleolus. Cyto-nuclear atypia and mitotic activity are usually discrete. In typical forms, the cells are scattered in a more or less abundant, usually myxoid, pattern. Foci of cartilaginous differentiation are exceptionally observed. The cellular component may be more or less dense. More cellular variants have been described, with sheet-like cellular arrangements (solid forms), but also pseudo-epithelial and pseudo-glandular forms. In pauci-cellular forms, the cells may be spindle-shaped, reminiscent of myofibroblast morphology. The myxoid matrix is stained with Alcian blue at $\mathrm{pH} 2.5$ and 1, a hyaluronidase resistant stain, suggesting the presence of chondroitin sulfates. Tumor cells always express vimentin, in about one third of cases S 100 protein (typically faint and focal staining), and often CD 99. Cytokeratin and EMA are sometimes expressed focally [18]. Other markers are not expressed, except sometimes NSE, synaptophysin, more rarely chromogranin, suggesting a possible neuroendocrine differentiation $[4,18]$. Sur le plan cytogénétique, le chondrosarcome myxoide extra squelettique est associé le plus souvent à une translocation chromosomique récurrente $t \quad(9 ; 22)$ (q22;q12), conduisant à un réarrangement génétique entre le gène EWS, localisé sur le chromosome 22, et le gène TEC, localisé sur le chromosome 9 [18]. Le réarrangement peut être recherché par RT-PCR sur tissu fixé. D'autres translocations sont plus rarement observées [18] : t(9;15) (q22; q12), t(9;17) (q22;q11.2), $\mathrm{t}(9 ; 17 ; 15)(\mathrm{q} 22 ; \mathrm{q} 11 ; \mathrm{q} 22), \mathrm{t}(2 ; 13)(\mathrm{q} 32 ; \mathrm{p} 12)$, et $\mathrm{t}(11 ; 22)$ (q11;p11).

Treatment involves primary surgery of the initial tumor and/or metastases, with unproven first-line radiation and chemotherapy. This tumor does not respond to chemotherapy and the results regarding radiotherapy are discordant [8].

The evolution is slow and extra skeletal myxoid chondrosarcoma is characterized by local aggressiveness, with fairly frequent local recurrences; nearly half of the patients have one or more local recurrences [19]. Survival is prolonged but the longterm prognosis is unfavorable (30-70\% survival at 10 years) $[14,20]$. Metastases are common (46-90\% of cases), often localized to the lungs, sometimes to soft tissue, bone, lymph nodes or brain. Currently, there are no well-established prognostic factors, most of them being controversial. The main poor prognostic factors reported are occurrence in males, late age of onset, tumor size greater than $10 \mathrm{~cm}$, proximal location, incomplete initial surgical excision, lack of resectability, and discovery of metastases at diagnosis [18, 21]. Histopronostic criteria such as necrosis, mitotic activity, degree of differentiation do not seem to influence the prognosis [8]. In fact, these different criteria are discordant in the literature and are mostly controversial [8].

\section{CONCLUSiON}

Extra skeletal myxoid chondrosarcoma is a rare soft tissue tumor. It is a tumor of very difficult and often delayed diagnosis; the diagnostic key is morphological, helped by immunohistochemistry and genetic study t $(9 ; 22)$. Despite its local aggressiveness, survival is prolonged and it is to be considered a lowgrade or intermediate-grade malignancy sarcoma.

\section{CONFLICTS OF INTEREST}

The authors declare no conflicts of interest.

\section{ACKNOWLEDGMENTS

Our sincere thanks to Professor
BENCHEKROUN NADIA

\section{REFERENCES}

1. McGrory, J.E., Rock, M.G., Nascimento, A.G., Oliveira, A.M. (2001). Extraskeletal Myxoid Chondrosarcoma. Clinical Orthopaedics and Related Research (1976-2007), 382:185-90.

2. Enzinger, F.M., Shiraki, M. (1972). Extraskeletal myxoid chondrosarcoma: An analysis of 34 cases. Human Pathology, 3(3):421-35.

3. Patel, S.R., Burgess, M.A., Papadopoulos, N.E., Linke, K.A., Benjamin, R.S. (1995). Extraskeletal myxoid chondrosarcoma. Long-term experience with chemotherapy. Am J Clin Oncol., 18(2); 161-3.

4. Okamoto, S., Hisaoka, M., Ishida, T., Imamura, T., Kanda, H., Shimajiri, S. (2001). Extraskeletal myxoid chondrosarcoma: A clinicopathologic, immunohistochemical, and molecular analysis of 18 cases. Human Pathology, 32(10); 1116-24.

5. Antonescu, C.R., Argani, P., Erlandson, R.A., Healey, J.H., Ladanyi, M., Huvos, A.G. (1998). Skeletal and extraskeletal myxoid chondrosarcoma. Cancer, 83(8); 1504-21.

6. Khouja, N., Amor, S.B., Jemel, H., Kchir, N., Boussen, H., Khaldi, M. (1999). Mesenchymal extraskeletal chondrosarcoma of the orbit: Report of a case and review of the literature. Surgical Neurology, 52(1):50-3.

7. Saleh, G., Evans, H.L., Ro, J.Y., Ayala, A.G. (1992). Extraskeletal myxoid chondrosarcoma. A clinicopathologic study of ten patients with longterm follow-up. Cancer, 70(12); 2827-30.

8. Saint-Blancard, P., Jancovici, R., Ceccaldi, B., Lagace, R., Sastre-Garau, X. (2006). Chondrosarcome myxoïde extrasquelettique du cou: à propos d'un cas avec métastases ganglionnaires locorégionales. La Revue de medecine interne, 2(27); 160-2. 
9. Acero, J., Escrig, M., Gimeno, M., Montenegro, T., Navarro-Vila, C. (2003). Extraskeletal myxoid chondrosarcoma of the infratemporal fossa: a case report. International Journal of Oral and Maxillofacial Surgery, 32(3); 342-5.

10. Algros, M.P., Collonge-Rame, M.A., Bedgejian, I., Tropet, Y., Delattre, O., Kantelip, B. (2003). Neurectodermal differentiation of extraskeletal myxoid chondrosarcoma: a classical feature? Ann Pathol., 23(3); 244-8.

11. Weiss, S.W. (1976). Ultrastructure of the so-called "chordoid sarcoma". Evidence supporting cartilagenous differentiation. Cancer, 37(1); 300-6.

12. Louvet, C., de Gramont, A., Krulik, M., Jagueux, M., Hubert, D., Brissaud, P. (1985). Extraskeletal mesenchymal chondrosarcoma: case report and review of the literature. JCO, 3(6); 858-63.

13. Mills, S.E., Frierson, H.F., Gaffey, M.J. (2000). Tumors of the Upper Aerodigestive Tract and Ear. Armed Forces Institute of Pathology, $476 \mathrm{p}$.

14. Nakashima, Y., Unni, K.K., Shives, T.C., Swee, R.G., Dahlin, D.C. (1986). Mesenchymal chondrosarcoma of bone and soft tissue. A review of 111 cases. Cancer, 57(12); 2444-53.

15. Salvador, A.H., Beabout, J.W., Dahlin, D.C. (1971). Mesenchymal chondrosarcomaobservations on 30 new cases. Cancer, 28(3); 605-15.
16. Harwood, A.R., Krajbich, J.I., Fornasier, V.L. (1981). Mesenchymal chondrosarcoma: a report of 17 cases. Clin Orthop Relat Res, (158):144-8.

17. Huvos, A.G., Rosen, G., Dabska, M., Marcove, R.C. (1983). Mesenchymal chondrosarcoma a clinicopathologic analysis of 35 patients with emphasis on treatment. Cancer, 51(7); 1230-7.

18. Oliveira, A.M., Sebo, T. J., McGrory, J.E., Gaffey, T.A, Rock, M.G., Nascimento, A.G. (2000). Extraskeletal Myxoid Chondrosarcoma: A Clinicopathologic, Immunohistochemical, and Ploidy Analysis of 23 Cases. Mod Pathol., 13(8):900-8.

19. Meis-Kindblom, J.M., Bergh, P., Gunterberg, B., Kindblom, L. G. (1999). Extraskeletal Myxoid Chondrosarcoma: A Reappraisal of Its Morphologic Spectrum and Prognostic Factors Based on 117 Cases. The American Journal of Surgical Pathology, 23(6); 636-50.

20. Guccion, J.G., Font, R.L., Enzinger, F.M., Zimmerman, L.E. (1973). Extraskeletal mesenchymal chondrosarcoma. Arch Pathol., 95(5):336-40.

21. Yagisawa, M., Ishitoya, J., Tsukuda, M., Sakagami, M. (2007). Chondrosarcoma of the temporal bone. Auris Nasus Larynx, 34(4); 527-31. 